\title{
INERTIA THEOREMS FOR MATRICES: THE SEMI-DEFINITE CASE
}

\author{
BY DAVID CARLSON AND HANS SCHNEIDER ${ }^{1}$ \\ Communicated by A. S. Householder, June 4, 1962
}

1. The inertia of a square matrix $A$ with complex elements is defined to be the integer triple In $A=(\pi(A), \nu(A), \delta(A))$, where $\pi(A)$ $\{\nu(A)\}$ equals the number of eigenvalues in the open right $\{$ left $\}$ half plane, and $\delta(A)$ equals the number of eigenvalues on the imaginary axis. The best known classical inertia theorem is that of Sylvester: If $P>0$ (positive definite) and $H$ is Hermitian, then $\operatorname{In} P H=\operatorname{In} H$. Less well known is Lyapunov's theorem [2]: There exists a $P>0$ such that $R(A P)=\frac{1}{2}\left(A P+P A^{*}\right)>0$ if and only if $\operatorname{In} A=(n, 0,0)$. Both classical theorems are contained in a generalization (Taussky [4], Ostrowski-Schneider [3]) which we shall call the

Main Inertia Theorem. For a given $A$, there exists a Hermitian $H$ such that $R(A H)>0$ if and only if $\delta(A)=0$. If $R(A H)>0$, then In $A=\operatorname{In} H$.

2.1. In this note we consider the case $\mathbb{R}(A H) \geqq 0$ which is far more complicated than the case $R(A H)>0$. We do not here solve the problem of all the possible relations of In $H$ to In $A$, except under additional assumptions.

THEOREM 1. Let $A$ be a given matrix for which all elementary divisors of imaginary eigenvalues are linear. If $H$ is a Hermitian matrix such that $\mathbb{R}(A H) \geqq 0$, then $\pi(H)=\pi, \nu(H)=\nu$ satisfy

$$
\pi \leqq \pi(A)+\delta(A), \quad \nu \leqq \nu(A)+\delta(A),
$$

respectively, and

$$
\text { rank } R(A H) \leqq \pi(A)+\nu(A) .
$$

Further, for any triple $(\pi, \nu, \delta)$ for which $\pi+\nu+\delta=n$, and $\pi, \nu$ satisfy (1), there exists an $H$ for which $R(A H) \geqq 0$ and In $H=(\pi, \nu, \delta)$. Thus (1) is in a sense the best possible inequality.

A more precise result may be proved if rank $\Omega(A H)=\pi(A)+\nu(A)$.

2.2. Theorem 2 concerns a matrix consisting of just one Jordan

1 The research of the authors was supported by the National Science Foundation under grant No. NSF G-19052 and by the United States Army under Contract No. DA-11-022-ORD-2059. 
block with one imaginary root. Its proof is largely computational. For assertion (4) (below) we use Cauchy's theorem on the separation of eigenvalues of a Hermitian matrix by the eigenvalues of a principal minor.

TheOREM 2. Let $A=\alpha I+U$, where $\alpha$ is pure imaginary and $U$ is the matrix with 1 in the first superdiagonal and 0 elsewhere. If $H$ is Hermitian of rank $r$ and $K=\mathbb{R}(A H) \geqq 0$ is of rank $s$, then

$$
2 s \leqq r,
$$

and for $\pi(H)=\pi, \nu(H)=\nu$,

$$
\begin{aligned}
& |\pi-\nu| \leqq 1, \\
& h_{i j}=0 \quad \text { if } i+j>r+1, \\
& k_{i j}=0 \quad \text { if } i>r / 2 .
\end{aligned}
$$

Again, the inequalities (3) and (4) are best possible, in the sense that if $r, s, \pi, \nu$, with $\pi+\nu=r$, are non-negative integers satisfying (3) and (4) then we can find an $H$ such that $R(A H) \geqq 0$, and $r=\operatorname{rank} H$, $s=\operatorname{rank} R(A H), \pi=\pi(H)$ and $\nu=\nu(H)$.

As a corollary of Theorem 2 we obtain a general existence theorem:

Corollary. For any matrix A, there exists a nonsingular Hermitian $H$ such that $R(A H) \geqq 0$.

In the special case that all elementary divisors of imaginary roots are linear, this result is known; cf. Givens [1].

2.3. Theorem 3. Let $A$ be a given matrix. If $H \geqq 0$ and $R(A H) \geqq 0$, then

$$
\text { rank } H \leqq \pi(A)+p(A),
$$

where $p(A)$ is the number of elementary divisors of imaginary roots. The inequality (7) is best possible.

Corollary 1. For a given matrix $A$, there exists an $H>0$ for which $\Re(A H) \geqq 0$ if and only if

$$
\nu(A)=0,
$$

(9) all elementary divisors of imaginary eigenvalues of $A$ (if any) are linear.

Corollary 2. If $R(A) \geqq 0$ and $H>0$ then all elementary divisors of imaginary eigenvalues of $A H$ are linear.

When $H=I$, Corollary 2 reduces to part of Theorem 2 of [3]. 
3.1. The proof of the Main Inertia Theorem hinges on the following lemma: If $\mathfrak{R}(A H)>0$, then $H$ is nonsingular. In this section we shall obtain a generalization of the Main Theorem by considering matrices with fixed null-space $\mathfrak{N}$. $\mathrm{By} \mathfrak{I}(A)$ we shall denote the nullspace of $A(x \in \mathfrak{N}(A): A x=0)$ and $\mathfrak{T}^{\perp}$ will be the orthogonal complement of $\mathfrak{x}\left(x \in \mathfrak{T}^{\perp}: y^{*} x=0\right.$ for all $\left.y \in \mathfrak{x}\right)$. Our results depend on the easily proved Theorem 4 which takes the place of the lemma quoted above.

We define In $A \leqq \operatorname{In} B$ if $\pi(A) \leqq \pi(B)$ and $\nu(A) \leqq \nu(B)(A, B$ need not be of the same order), and $\operatorname{In} A=\operatorname{In} B$ if $\pi(A)=\pi(B)$ and $\nu(A)$ $=\nu(B)$.

TheOREM 4. If $\Re(A H) \geqq 0$ then

$$
\begin{aligned}
\Re(\Re(A H)) & \supseteq \Re(H), \\
A \Re(H)^{\perp} & \subseteq \Re(H)^{\perp}, \\
\operatorname{In}\left(A \mid \Re(H)^{\perp}\right) & \leqq \operatorname{In} H .
\end{aligned}
$$

Here $A \mid \Re(H)^{\perp}$ is the restriction of $A$ to $\Re(H)^{\perp}$.

As an immediate corollary to the proposition we have

Corollary. If $\Re(A H) \geqq 0$ and In $\left(A^{*} \mid \mathfrak{T}(H)\right)=(0,0, \delta)$ then

$$
\text { In } A=\operatorname{In}\left(A \mid \mathfrak{T}(H)^{\perp}\right) \leqq \operatorname{In} H .
$$

In particular if $\mathbb{R}(A H) \geqq 0$ and $H$ is nonsingular, then $\operatorname{In} A \leqq \operatorname{In} H$.

3.2. It is interesting to note that in our next theorem, the inequalities will go in the opposite direction. This theorem reduces to the Main Inertia Theorem when $\mathfrak{X}=(0)$.

Theorem 5. Let $\mathfrak{x}$ be a subspace of $V$. There exists a Hermitian $H$ such that

$$
\Re(A H) \geqq 0,
$$

and

$$
\Re(\Re(A H))=\mathscr{N}(H)=\Re .
$$

if and only if

$$
A \Re^{\perp} \subseteq \Re^{\perp}
$$

and

$$
\delta\left(A \mid \Re^{\perp}\right)=0 .
$$

If (13) and (14) hold, then

$$
\text { In } H=\operatorname{In}\left(A \mid \Re^{\perp}\right) \leqq \operatorname{In} A .
$$


Corollary 1. Let $A$ and $\Re$ satisfy conditions (15) and (16). If

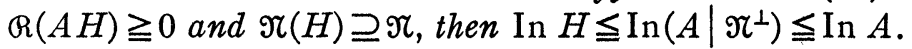

Corollary 2. If $\delta(A)=0$ and $R(A H) \geqq 0$, then In $H \leqq \operatorname{In} A$. If, in addition, $\delta(H)=0$ (i.e., $H$ is nonsingular), then $\operatorname{In} H=\operatorname{In} A$.

Corollary 3. If $\mathbb{R}(A H) \geqq 0$ and $\operatorname{rank} R(A H)=\operatorname{rank} H=\pi(A)$ $+\nu(A)$, then, again, In $H=\operatorname{In} A$.

3.3. Suppose the conditions of Theorem 5 are fulfilled and there exists a $K$ such that $\mathfrak{R}(A K) \geqq 0$, and $\mathfrak{T}(\mathfrak{R}(A K))=\mathfrak{N}(K)=\Re, A$ and $\Re$ being given. When does every $H$ satisfying $\Re(R(A H))=\Re$ (and not necessarily satisfying $\mathbb{R}(A H) \geqq 0)$ also satisfy $\mathfrak{\Re}(H)=\mathfrak{N}$ ? For $\Re=(0)$, the question is: When does $R(A H)=0$ imply $H=0$ ?. The conditions for this are well-known (Corollary below). Thus our Theorem 6 is a generalization of the known Corollary 6.

We require the following definition. If $A$ and $B$ are square matrices (possibly of different orders), we let

$$
T(A, B)=\prod_{i, j}\left(\alpha_{i}+\beta_{j}\right)
$$

the product being taken over all pairs of eigenvalues $\left(\alpha_{i}, \beta_{j}\right)$ of $A$ and $B$, and for the sake of convenience we write $T(A)=T\left(A, A^{*}\right)$. If $A$ is the empty matrix (an operator on a 0 -dimensional space), certain consistency conditions force us to take $T(A, B)=1$.

TheOREM 6. Let $\mathfrak{T}$ be a subspace of $V$, and $A$ a matrix for which $A \mathfrak{N}^{\perp} \subseteq \mathfrak{N}^{\perp}$. If

$$
T\left(A\left|\Re^{\perp}, A^{*}\right| \Re\right) \cdot T\left(A^{*} \mid \Re\right) \neq 0
$$

then $\Re(R(A H)) \supseteq \Re$ implies $\Re(H) \supseteq \Re$. Conversely, if

$$
T\left(A\left|\Re^{\perp}, A^{*}\right| \Re\right) \cdot T\left(A^{*} \mid \Re\right)=0
$$

then there exists a Hermitian $H$ such that $\mathfrak{N}(\mathbb{R}(A H)) \supseteq \Re$ but $\mathfrak{N}(H) \supseteq \Re$.

CoROllary 1. There exists a nonzero $H$ such that $R(A H)=0$ if and only if $T(A)=0$.

Corollary 2. Let $\mathbb{R}(A H) \geqq 0$ and let $\mathfrak{N}=\mathfrak{N}(\mathcal{R}(A H))$. If $A \mathfrak{T}^{\perp} \subseteq \mathfrak{N}^{\perp}$ and (17) holds then $\Re(\Re(A H))=\Re(H)$.

Corollary 3. Let $\mathbb{R}(A K) \geqq 0$ and $\mathfrak{N}=\mathfrak{\Re}(K)=\mathfrak{\Re (}(\mathcal{R}(A K))$. If (17) holds, then $\mathbb{R}(A H) \geqq 0$ and $\mathfrak{N}(\mathbb{R}(A H))=\mathfrak{N}$ implies that $\mathfrak{N}(H)=\mathfrak{N}$. Conversely if (18) holds, then there exists a Hermitian $H$ such that $\mathfrak{R}(A H) \geqq 0$ and $\mathfrak{N}(\mathcal{R}(A H))=\mathfrak{N}$ but $\mathfrak{N}(H)$ is properly contained in $\mathfrak{N}$. 
4. As in [3], the matrix $A$ is called $H$-stable if, for Hermitian matrices $H$, In $A H=(n, 0,0)$ if and only if $H>0$. A necessary and sufficient condition for $H$-stability was found in [3], Theorem 4. However, this condition does not greatly facilitate the determination of $H$-stability for a given matrix $A$. Our Theorem 7 below provides an effective test for $H$-stability. The only candidates are nonsingular $A$ with $\mathcal{R}(A) \geqq 0$, and thus we need merely diagonalize $\mathbb{R}(A)$ and examine the transform of $\mathfrak{g}(A)=(1 / 2 i)\left(A-A^{*}\right)$.

THeORem 7. Let $A$ be a nonsingular matrix with $R(A) \geqq 0$, and let $k=\max _{H>0} \delta(A H)$. Let $S$ be any nonsingular matrix for which $S^{*} A S$ $=A^{\prime}=P+i Q$, where $P=P_{11} \oplus 0$ and $Q$ are Hermitian, and $P_{11}>0$. If $Q$ is partitioned conformably with $P$, then rank $Q_{22}=k$. In particular, $A$ is $H$-stable if and only if $Q_{22}=0$.

CoRollary. If $A$ is an $H$-stable matrix of order $n$, then rank $\mathbb{R}(A)$ $\geqq n / 2$.

\section{REFERENCES}

1. W. Givens, Elementary divisors and some properties of the Lyapunov mapping $X \rightarrow A X+X A^{*}$, Argonne National Laboratory Report ANL-6456, 1961.

2. A. Lyapunov, Probléme géneral de la stabilitê du mouvement, Comm. Soc. Math. Kharkov (1892); Annals of Mathematics Studies, No. 17, Princeton Univ. Press, Princeton, N. J., 1947.

3. A. M. Ostrowski and H. Schneider, Some theorems on the inertia of general matrices, J. Math. Anal. Appl. 4 (1962), 72-84.

4. O. Taussky, A generalization of a theorem by Lyapunov, J. Soc. Indust. Appl. Math. 9 (1961), 640-643.

UNIVERSITY OF WISCONSIN 